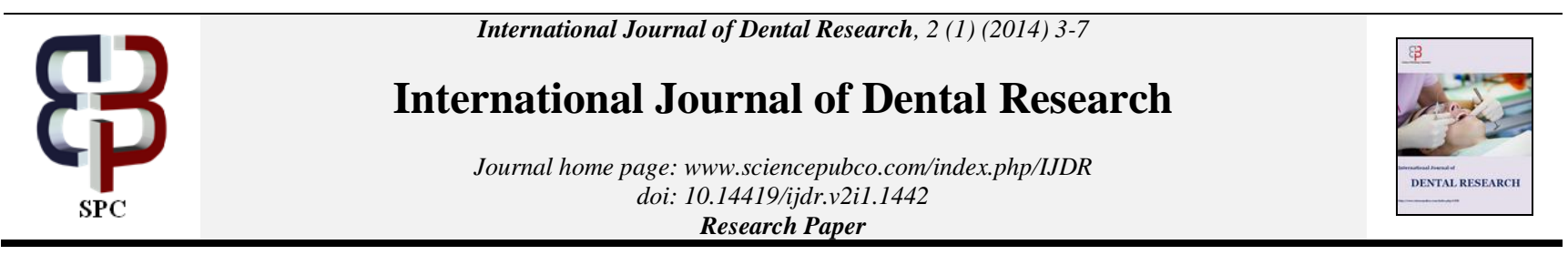

\title{
Histochemical evaluation of stromal components in fibrous dysplasia, central ossifying fibroma and juvenile ossifying fibroma affecting the jaw bones
}

\author{
Parul Maheshwari *, Nirmala N Rao, Raghu Radhakrishna \\ Manipal College of Dental Sciences Manipal University, Udupi District \\ *Corresponding author E-mail: docparulmaheshwari@gmail.com
}

\begin{abstract}
Background: Oral Pathologists often come across fibro-osseous lesions involving the jaws with intersecting clinico-pathologic features which makes their diagnosis challenging. Due to the difference in their pathogenesis, the treatment varies and so does the prognosis which makes differentiation essential.

Objectives: This study intended to evaluate the stromal components of fibrous dysplasias, central and juvenile ossifying fibromas affecting the jaw bones using histochemical stains and to determine the histological parameters important for their differentiation.

Methods: Three sections each of forty-seven formalin-fixed decalcified paraffin-embedded tissues were stained with three special stains (Modified Tetrachrome, Modified Attwood's and Potassium-monopersulfate aldehyde fuschin stain) and analysed for morphology and structure of calcifications, osteoblastic rimming, cellularity and vascularity of the stroma, collagen pattern and oxytalan fibers.

Results: Despite the overlap in the histopathological features, few parameters were more pertinent to one lesion than the others. Fibrous dysplasia cases had characteristic thin curvilinear calcifications which were discontinuous whereas central and juvenile ossifying fibromas had broader calcifications which were interconnected. The structure of calcifications was mostly woven in fibrous dysplasia but few longstanding cases did show lamellar bone. The cellularity and vascularity was considerably higher in ossifying fibromas than fibrous dysplasia. Oxytalan fibers were more evident in cases of ossifying fibromas.

Conclusion: Histopathological analysis alone may not suffice for the diagnosis of fibro-osseous lesions due to the apparent similarity, thus stressing on the importance of radiologic and genetic analysis in the diagnosis of fibro-osseous lesions.
\end{abstract}

Keywords: Fibro-osseous lesions, fibrous dysplasia, histochemical, ossifying fibroma, special stains.

\section{Introduction}

The term "fibro-osseous lesions" (FOLs) has gained wide acceptance as a general designation for a challenging group of pathologic conditions that often pose difficulty in diagnosis. Common to all these pathologic entities is the replacement of normal bone architecture by a benign fibrous tissue composed of fibroblasts and collagen, consisting of varying amounts of mineralized material. Categorization of the fibro-osseous lesions depends on correlation of the patient's history, clinical findings, radiographic criteria, and histopathology. However, because of overlapping clinical, radiological and histological features, their classification has evoked much discussion.

Amongst the broad category of FOLs, some lesions are considered neoplastic while others are developmental or reactive in nature. Juvenile ossifying fibroma (JOF) and central ossifying fibroma (COF) are considered true neoplastic lesions, while fibrous dysplasia (FD) and cemento-osseous dysplasia (COD) are said to be developmental and reactive lesions respectively (Nwizu et al. 2010). Central ossifying fibroma and fibrous dysplasia of the jaws are the two most common fibro-osseous lesions which may be associated with significant cosmetic and functional disturbances. It is important to distinguish the two as they show distinct patterns of disease progression.
Routine histological procedures pose a difficulty in distinguishing osteoid from woven bone in decalcified, routinely stained preparation of bone. However, obvious distinction between osteoid, woven and lamellar bone is possible with special staining and this facilitates the study of fibro-osseous lesions. It also has the advantage of displaying simultaneously irregularities in the mineralized matrix and morphology of bone cells (Ralis \& Watkins 1992).

This study is an attempt to compare the stromal components of fibrous dysplasia, central and juvenile ossifying fibroma affecting the jaw bones using special stains in order to identify the histological features which are more pertinent to one condition than the other and may facilitate their differentiation in correlation with their clinical and radiographic features.

\section{Materials and methods}

Study sample included a total of forty-seven formalin fixed, decalcified, paraffin embedded tissue blocks which included eighteen cases of fibrous dysplasia, nineteen cases of central ossifying fibroma and seven cases of juvenile ossifying fibroma. One case each of peripheral ossifying fibroma, normal bone tissue and one tissue block of fracture callus were used as a control. The diagno- 
sis of these cases was based on clinical, radiographical and histopathological criteria using hematoxylin and eosin staining.

Three sections were prepared from each tissue block at $5 \mu \mathrm{m}$ thickness using a semi-automatic rotary microtome. Sections were deparaffinized in xylene and hydrated through decreasing grades of alcohol and stained with Modified tetrachrome stain (Ralis \& Watkins 1992), Modified Attwood's stain (Putns \& Desa 1977) and Potassium monopersulfate-aldehyde fuchsin stain for oxytalan fibers. The cases were then evaluated for seven histological parameters which included the morphology and structure of calcifications, osteoblastic rimming of the calcifications, arrangement of collagen fibers in the stroma, cellularity and vascularity of the stroma; and the presence of oxytalan fibers. All these parameters were categorized and a pictorial template prepared to reduce the subjectivity in interpretation of the parameters amongst the observers. The categories were given scores for further evaluation. Statistical analysis was done using SPSS version 16 and the findings obtained by the two observers were subjected to Kappa statistical test to check for inter-observer agreement. Further, Chisquare test was applied to analyze the statistical significance.

\section{Results and observations}

Modified Tetrachrome stain was found to be useful in distinguishing woven bone from osteoid in decalcified paraffin-embedded sections which at times may be difficult in routine staining (Fig 1). Modified Attwood's stain also stained woven bone, osteoid and lamellar bone analogous to the structures stained by Modified Tetrachrome stain facilitating the identification of the structure of bone in decalcified sections. Potassium monopersulfate-aldehyde fuchsin stained oxytalan fibers dark blue in contrast to the light green collagen fibers

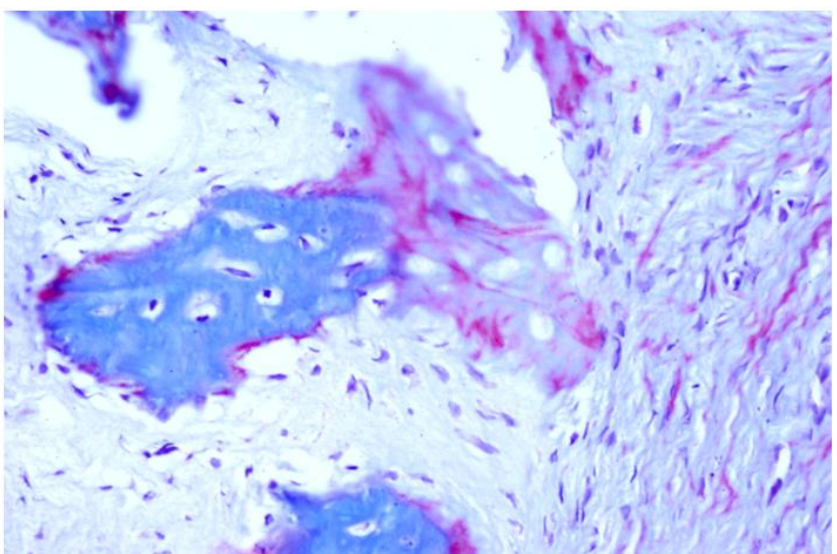

Fig. 1: Showing areas of woven bone (dark blue) and osteoid (light blue with pink patches) in control tissue stained with Modified tetrachrome stain $(40 x)$

Evaluation of morphology of calcifications revealed that eleven out of eighteen cases of fibrous dysplasia had thin curvilinear or Chinese letter shaped trabeculae which were disconnected (Fig 2). Five cases showed broad curvilinear trabeculae and two cases had trabeculae which were in the form of a lattice. One case of fibrous dysplasia also showed calcifications which resembled spherules in addition to thin curvilinear trabeculae. On the other hand, central ossifying fibroma cases showed broad curvilinear trabeculae in eleven out of nineteen cases, while in four cases they were connected to form lattice. Fourteen out of nineteen cases also showed presence of spherules. Four cases of juvenile ossifying fibroma presented with calcifications in the form of broad curvilinear trabeculae, two in the form of lattice and four cases showed presence of spherules (Table 1).

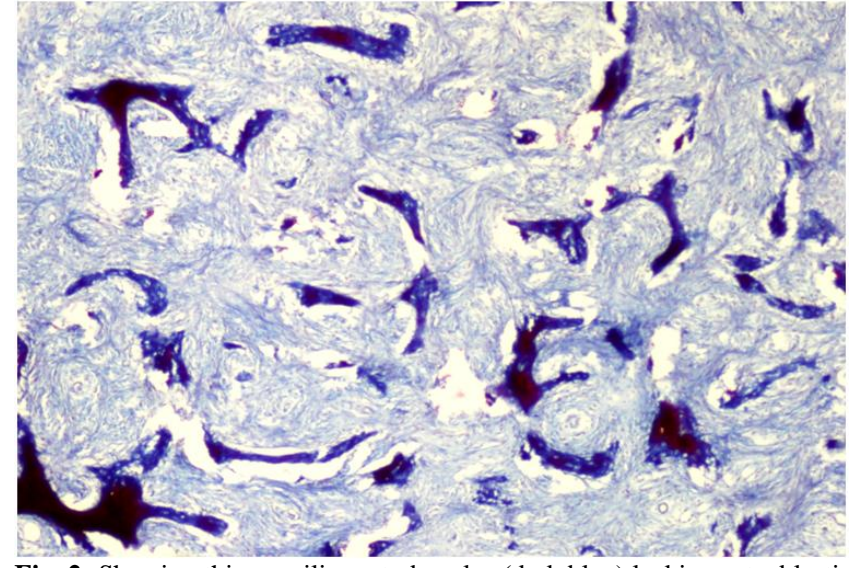

Fig. 2: Showing thin curvilinear trabeculae (dark blue) lacking osteoblastic rimming with low stromal cellularity and vascularity and loose collagen arrangement seen in FD stained with Modified tetrachrome stain (10x).

Table 1: Comparison of morphology, structure and osteoblastic rimming of calcifications between different lesions.

\begin{tabular}{llllll}
\hline Parameter & \multicolumn{2}{l}{ Group } & & p-value & Significance \\
\hline Morphology & FD & COF & JOF & & \\
Total number of cases & 18 & 19 & 7 & & \\
Thin and Curvilinear & 11 & 0 & 0 & & \\
Broad \& Curvilinear & 5 & 11 & 4 & 0.001 & \\
Lattice & 2 & 4 & 2 & & - \\
Spherules & 1 & 14 & 4 & & \\
Structure & FD & COF & JOF & & \\
Woven & 11 & 12 & 6 & & \\
Lamellar & 7 & 3 & 0 & & \\
Globular Acellular & 2 & 11 & 4 & 0.002 & - \\
Globular Cellular & 0 & 2 & 2 & & \\
Osteoblastic rimming & FD & COF & JOF & & \\
Present & 5 & 13 & 3 & 0.019 & - \\
Absent & 13 & 6 & 4 & & \\
\hline
\end{tabular}

$\mathrm{P}$ value $<0.001-$ Significant

Next, the structure of the calcifications was evaluated. It was observed that eleven out of eighteen fibrous dysplasia cases contained woven bone while seven cases showed lamellar bone. Besides woven and lamellar bone, globular acellular calcifications which were dark pink in the center with a dark blue periphery and lacked osteocytes were seen in two cases. However, central ossifiying fibroma cases showed woven bone in twelve cases and lamellar bone in three cases.

Also globular calcifications were seen, which were acellular in eleven cases and cellular in two cases. Juvenile ossifying fibroma cases showed presence of woven bone or globular acellular calcifications but not lamellar bone (Fig 3).

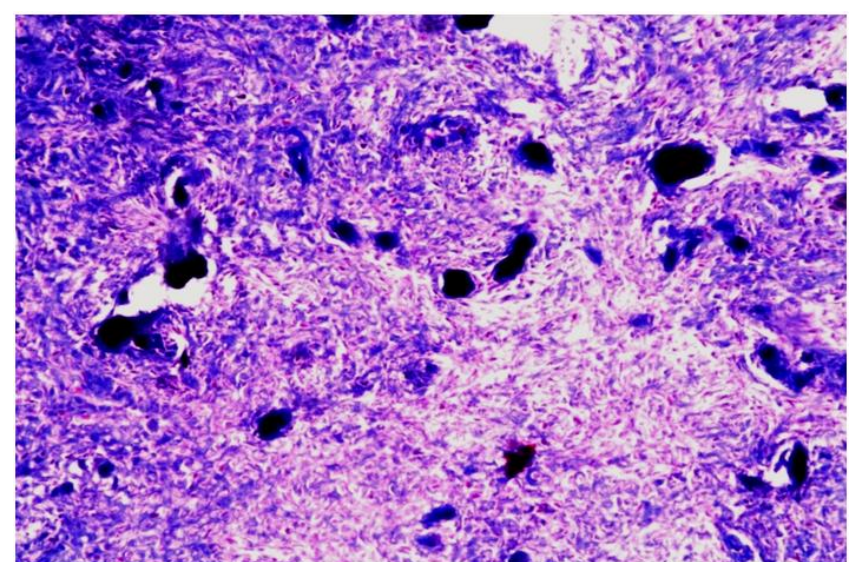

Fig. 3: Showing areas of globular acellular calcifications (dark blue) with high cellularity seen in a case of JPOF stained with Modified tetrachrome stain $(4 x)$.

The calcifications were also evaluated for the presence of osteoblastic rimming. Thirteen out of eighteen cases of fibrous 
dysplasia lacked osteoblastic rimming while it was present in thirteen out of nineteen cases of central ossifying fibroma.

The cellularity of the stroma was evaluated by counting the number of cells lying on the meridian line of the grid reticule at high power. It was observed that stromal cellularity varied significantly ( $\mathrm{p}$ value $<0.01$ ) between fibrous dysplasia with mild to moderate cellularity, central ossifying fibroma and juvenile ossifying fibroma cases with moderate to severe cellularity (Table 2). Vascularity of the stroma was categorized as less or more based on the number of blood vessels present per 3 high power fields (HPF). Fibrous dysplasia cases showed less vascularity in eleven out of eighteen cases while central ossifying fibroma cases showed more vascularity in twelve out of nineteen cases. Juvenile ossifying fibroma cases had more vascularity in five out of seven cases (Table 2). On analyzing collagen fiber arrangement, fibrous dysplasia cases mainly had a parallel or loose arrangement, while central ossifying fibroma cases and juvenile ossifying fibroma cases predominantly showed whorled arrangement of collagen fibers (Table 2).

The presence of oxytalan fibers in the connective tissue was observed in seventeen out of nineteen cases of central ossifying fibroma (Fig 4), five out of seven cases of juvenile ossifying fibroma though only in five out of eighteen cases of fibrous dysplasia. Out of all the parameters cellularity and presence of oxytalan fibers differed significantly between the lesions (Table 2).

Table 2: Comparison of stromal cellularity, vascularity, collagen pattern and presence of oxytalan fibers between different lesions.

\begin{tabular}{llllll}
\hline Parameter & \multicolumn{2}{l}{ Morphology } & & p-value & Significance \\
\hline Cellularity & FD & COF & JOF & & \\
Total number of & 18 & 19 & 7 & & \\
cases & 13 & 0 & 0 & $<0.001$ & \\
Mild & 5 & 9 & 2 & & Significant \\
Moderate & 0 & 10 & 5 & & \\
Severe & FD & COF & JOF & & \\
Vascularity & 11 & 7 & 2 & & \\
Less & 7 & 12 & 5 & 0.032 & - \\
More & FD & COF & JOF & & \\
Collagen Pattern & 1 & 8 & 3 & & \\
Whorled & 7 & 7 & 0 & & \\
Parallel & 4 & 4 & 3 & 0.008 & - \\
Random & 6 & 0 & 1 & & \\
Loose & FD & COF & JOF & & \\
Oxytalan fibers & 5 & 17 & 5 & $<0.001$ & Significant \\
Present & 13 & 2 & 2 & & \\
Absent & & & & &
\end{tabular}

$\mathrm{P}$ value $<0.001-$ Significant

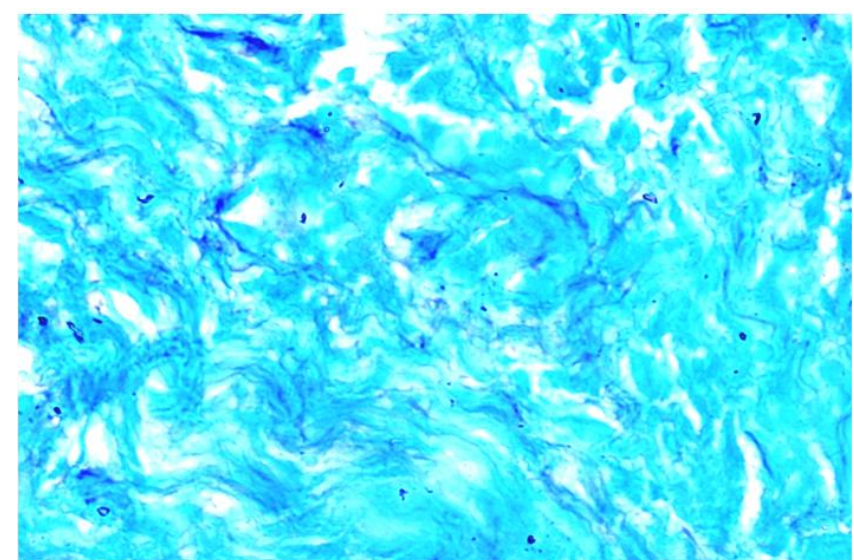

Fig. 4: Showing oxytalan fibers (dark purple) interwoven with collagen fibers (light green) in the stroma of COF stained with Potassium monopersulphate aldehyde fuchsin stain (40x).

\subsection{Scoring Criteria}

Each parameter was allotted a score (Table 4) and a total score for each case was calculated. Each category of morphology of calcification was allotted a score of 5 as they were independent of each other. In structure of calcification woven bone was allotted a score of 5, whereas lamellar bone was allotted 10 as it follows only after formation of woven bone. Globular acellular and cellular calcifications were allotted a score of 5. Presence of osteoblastic rimming of the calcifications was allotted a score of 5 whereas absence was given a score of 0 . Score of 5 was given for mild stromal cellularity, 10 for moderate and 15 for severe stromal cellularity. Vascularity was scored 5 if it was less and 10 if it was more. All collagen patterns were given a score of 5. Presence of oxytalan fibers was given a score of 5 , while its absence was given 0 score. It was observed that score for fibrous dysplasia was less than 45 whereas it was more than or equal to 45 in central ossifying and juvenile ossifying fibroma cases.

Table 3: Scores for parameters

\begin{tabular}{llll}
\multicolumn{4}{c}{ Table 3: Scores for parameters } \\
\hline Parameter & Score & Parameter & Score \\
\hline Morphology of calcification & & Cellularity & \\
Thin curvilinear & 5 & Mild & 5 \\
Broad curvilinear & 5 & Moderate & 10 \\
Lattice & 5 & Severe & 15 \\
Spherules & 5 & & \\
Structure of calcification & & Vascularity & \\
Woven & 5 & Less & 5 \\
Lamellar & 10 & More & 10 \\
Globular acellular & 5 & & \\
Globular cellular & 5 & Collagen Pattern & \\
& & Whorled & 5 \\
Osteoblastic rimming & & Parallel & 5 \\
Present & 5 & Random & 5 \\
Absent & 0 & Loose & 5 \\
& & Oxytalan fibers & \\
& & Present & 5 \\
& & Absent & 0 \\
\hline
\end{tabular}

\section{Discussion}

Amidst the overlapping presentations of many fibro-osseous lesions, fibrous dysplasias of the jaws are the hardest to differentiate from central ossifying fibroma in the same location. Although some criteria namely presence of woven bone, peripheral osteoblastic rimming, and curvilinear pattern of bone have been laid down for the diagnosis of fibrous dysplasia, but their diagnostic significance has been questioned. Most of these criteria have been adapted from the fibrous dysplasia lesions affecting the long bones. However, the embryological difference in the origin of the craniofacial bones has distinguished lesions in the craniofacial region from those occurring in other parts of the body and hence the two lesions cannot be totally equated. Need of the hour is to validate or renew the criteria for the differentiation of the fibroosseous lesions of the jaw.

The morphology of the calcified component was noticeably different in each of the lesions. Significant number of fibrous dysplasia presented with thin curvilinear trabeculae which were disconnected whereas central ossifying fibroma and juvenile ossifying fibroma cases mostly presented with broad curvilinear trabeculae or spherules. A possible explanation for the variation in morphology could be the difference in expression of osteocalcin, a bone matrix protein between FD and COF which has been reported by many authors [Toyosawa et al. (2007), Hopyan et al. (1999)]. Osteocalcin, is secreted by fully differentiated osteoblasts and osteocytes and is functional in inhibiting crystal growth and enhancing recruitment of osteoclasts. Hopyan et al. (1999) have reported a higher expression of osteocalcin in fibrous dysplasia cases when compared to osteosarcoma and myositis ossificans. They concluded that the lack of osteocalcin is associated with an increase in bone of improved functional quality in the absence of the effect of osteocalcin on the number of osteoblasts, bone resorption or mineralization. He concluded that osteocalcin is thought to be a negative regulator of bone formation and may be responsible for the disconnected appearance of trabeculae in fibrous dysplasia by inhibition of bone formation.

The structure of calcifications also differed between the lesions. Though fibrous dysplasia cases mostly contained woven bony 
trabeculae, few cases showed lamellar bone formation. This observation is also in agreement with Waldron (1960) and others who have reported lamellar bone formation in lesions that otherwise met the clinical and radiologic criteria of fibrous dysplasia. Therefore the presence of lamellar bone cannot rule out fibrous dysplasia as a diagnosis. To reason the presence of lamellar calcifications in fibrous dysplasia requires a look into its pathogenesis. The certainty of the mosaic nature of this lesion having both mutated and non-mutated osteoblasts was confirmed by Riminucci et al. (2006). They showed that pure strains of mutated cells from Fibrous Dysplasia would fail to generate fibrous dysplasia tissue in the mouse as the mutated cells were less viable and were eventually lost and replaced by normal osteoblasts in the long standing lesions. This was an important study as it gave an explanation as to why long standing lesions of FD may present with lamellar bone. Riminucci et al. (2006) further proposed that normal osteoblasts which undergo full differentiation may remodel the bone trabeculae and rim the calcifications. Thus, presence of osteoblastic rimming cannot exclude the diagnosis of FD Osteoblastic rimming was absent in most but not all of our cases of fibrous dysplasia. On the contrary it was present in majority of the central ossifying fibroma cases.

Riminucci et al. (2006) also observed that different clinical lesions of FD include different proportions of assayable mutated cells and they seem to decline progressively with age and may ultimately disappear. Hence, though there is a maturation arrest of the pathological bone in fibrous dysplasia due to the mutated osteoblasts which fail to undergo complete differentiation, there is a possibility of it getting replaced by the lamellar bone with the ageing of the lesion. However, the presentation of calcifications in different lesions is directly dependent on the relative frequency of the mutated and non-mutated osteoblasts. If the latter concept is assumed to be true it can be accepted that in contrast to extragnathic fibrous dysplasia lesions, gnathic fibrous dysplasia lesions show tendency towards maturation. The difference in maturation between the fibrous dysplasia of long bones and that of craniofacial bones is thought to be due to the different inductive influences during development of these embryologically distinct types of bones. Marie et al have showed that the fraction of osteoid surface with morphologically mature osteoblasts was very low in fibrous dysplasia compared with the normal bone (Marie et al. 1997). Further, the artefactual separation of the connective tissue stroma from the calcification may lead to difficulty in discerning the rimming in some of central ossifying fibroma lesions. In the present study also, lamellar calcifications were found in the cases of FD which belonged to lesions of long standing duration. Similar cases have also been reported by Eversole et al where woven bone was replaced by lamellar bone seen on a subsequent biopsy within an interval of two years in pre-pubertal age group individuals (Eversole et al. 1972).

In the present study, spherical calcifications or spherules were observed in the central ossifying fibroma cases and in two cases of fibrous dysplasia. These were acellular round to oval structures of various sizes with darker staining than others. Boysen et al. (1979) have also reported the presence of spheroid calcifications more in COF cases than in FD cases. However, Sisson et al. (1993) reported the presence of calcified spherules in cases of extragnathic fibrous dysplasia and suggested that presence of calcified spherules is not suggestive of diagnosis of cementifying fibroma or of odontogenic origin. The true nature of these calcifications cannot be determined by light microscopy therefore it is difficult to say whether they are bone, cementum, metaplastic bone or dentin [Sisson et al. (1993), Burkhardt (1989)].

According to the present study, cellularity of stroma ( $p$ value $<0.001)$ is a significant parameters in differentiating fibrous dysplasia from central ossifying fibroma and juvenile ossifying fibroma. Our results were in agreement with the reports of other authors that cases of fibrous dysplasia usually exhibit mild to moderate cellularity. The cellularity of the stroma decreases in old fibrous dysplasia cases with a relative increase in collagen arranged in a parallel fashion and has a rather monotonous distribu- tion [Nwizu et al. (2010), Neville 2009 p639]. Sakamoto et al have also reported lower cellularity in older cases of fibrous dysplasia in comparision to early cases with a statistical significance (Sakamoto et al. 2005). The reason for decrease in cellularity can be attributed to the decrease in frequency of mutated cells which have a higher proliferation rate compared to normal cells (Riminucci et al. 2006). In contrast, the central ossifying fibroma and juvenile ossifying fibroma lesions show moderate to severe cellularity and contain dense collagenous stroma arranged in whorls with comparatively more vascularity.

In the present study oxytalan fibers were observed in all but two cases of ossifying fibroma and in five cases of fibrous dysplasia. Our findings indicate that presence of oxytalan fibers cannot be correlated with odontogenic origin or with any particular FOL as they were also found in fibrous dysplasia cases as well which is considered non-odontogenic. Hamner \& Fullmer (1966) also found oxytalan fibers in most fibro-osseous lesions of the jaw including fibrous dysplasia, provided that mature collagen was present. Also, Ono et al. (2007) found oxytalan fibers in only 50\% of their cases and concluded that ossifying fibroma does not always originate from the periodontal tissue, even if they are near the alveolar bone area. Thus, presence of oxytalan fibers cannot be used as a criterion to differentiate fibrous dysplasia from central ossifying fibroma and juvenile ossifying fibroma. Thus, histopathology alone may not suffice the diagnosis of fibro-osseous lesions due to the varied presentation depending on the stage of the lesion.

The present study also proposes a scoring criterion which may help in differentiating fibrous dysplasia from central ossifying fibroma and juvenile ossifying fibroma of the jaws in a more objective way. According to this criteria, a unique score was allotted to each category or histological presentation of the seven parameters (morphology of calcifications, structure of calcifications, osteoblastic rimming of calcifications, cellularity of stroma, vascularity of stoma, collagen pattern of the stroma and presence of oxytalan fibers), and the total of all the scores for a particular case was calculated. It was found that above criteria clearly differentiated fibrous dysplasia with a score less than 45 from central ossifying fibroma and juvenile ossifying fibroma with score more than or equal to 45 in all the forty-four cases. Nonetheless, these scoring criteria require further validation by application on a larger sample size and lesions of different stages.

\section{Conclusion}

The histopathological characteristics may aid towards the diagnosis of FOL, however it should not be based on any single criteria. The dynamic nature of these lesions requires correlation with clinical, radiographic features and genetic analysis.

\section{Acknowledgement}

I would like to thank my family and teachers for their incessant support through this study and every phase of life.

\section{References}

[1] Nwizu NN, Aguirre A, Chen F (2010) Diagnostic challenges of benign fibro-osseous lesions and psammomatous meningiomas of the craniofacial region: a comparative review of their clinico-pathological features. North American Journal of Medical Sciences. 3(1), 17-23.

[2] Ralis ZA, Watkins G (1992) Modified tetrachrome method for osteoid and defectively mineralized bone in paraffin sections. Biotechology Histochemistry. 67(6), 339-45.

[3] Putns S, Desa DJ (1977) Application of a modified Attwood's stain to the study of decalcified bone sections. Journal of Clinical Pathology. 30(9), 900-3

[4] Toyosawa S, Yuki M, Kishino M, Ogawa Y, Ueda T, Murakami S, Konishi E, lida S, Kogo M, Komori T, Tomita Y (2007) Ossifying fibroma vs fibrous dysplasia of the jaw: molecular and immunological characterization. Modern Pathology. 20(3), 389-96. 
[5] Hopyan S, Gokgoz N, Bell RS, Andrulis IL, Alman BA, Wunder JS (1999) Expression of osteocalcin and its transcriptional regulators core-binding factor alpha 1 and MSX2 in osteoid-forming tumours. Journal of Orthopaedic Research. 17(5), 633-8.

[6] Waldron CA. (1970) Fibro-osseous lesions of the jaw. Journal of Oral Surgery. 28, 58-64.

[7] Riminucci M, Saggio I, Robey PG, Bianco P. (2006) Fibrous dysplasia as a stem cell disease. Journal of Bone and Mineral Research. 21(2), 125-31.

[8] Marie PJ, de Pollak C, Chanson P, Lomri A. (1997) Increased proliferation of osteoblastic cells expressing the activating Gs $\alpha$ mutation in monostotic and polyostotic fibrous dysplasia. American Journal of Pathology. 150(3), 1059-69.

[9] Eversole LR, Sabes WR and Rovin S. (1972) Fibrous dysplasia: A nosologic problem in the diagnosis of fibro 70 osseous lesions of the jaws. Journal of Oral Pathology. 1(5), 189-220.

[10]Boysen ME, Olving JH, Vatne K, Koppang HS. (1979) Fibro-osseous lesions of the cranio-facial bones. Journal of laryngology and Otology. 93,793-807.

[11] Sissons HA, Steiner GC, Dorfman HD. (1993) Calcified spherules in fibro-osseous lesions of bone. Archives of Pathology and Laboratory Medicine. 117(3), 284-90.

[12]Burkhardt A. (1989) Dentin formation in so called "fibro-osteocemental" lesions of the jaws: histologic, electron microscopic and immunohistochemical investigations. Oral Surgery Oral Medicine Oral Pathology. 68, 729-38

[13]Neville BW, Damm DD, Allen CM, Bouquot JE (2009) Bone Pathology. Oral and Maxillofacial Pathology 3rd edition. WB Saunders Company.

[14]Sakamoto A, Chen M, Nakamura T, Xie T, Karsenty G, Weinstein LS. A comparative study of fibrous dysplasia and osteofibrous dysplasia with regard to Gsalpha mutation at the Arg201 codon: polymerase chain reaction-restriction fragment length polymorphism analysis of paraffin-embedded tissues. Journal of Biology and Chemistry. 2005 Jun $3 ; 280(22): 21369-75$.

[15]Hamner JE 3rd, Fullmer HM. Oxytalan fibers in benign fibro-osseous jaw lesions. Archives of Pathology. 1966 Jul; 82(1):35-9.

[16] Ono A, Tsukamoto G, Nagatsuka H, Yoshihama Y, Rivera RS, Katsurano M, Yao M, Sasaki A. An immunohistochemical evaluation of BMP-2, -4, osteopontine, osteocalcin and PCNA between ossifying fibromas of the jaws and peripheral cement-ossifying fibromas on the gingival. Oral Oncology. 2007 Apr; 43(4):339-44 